\title{
What Is Brain Mapping?
}

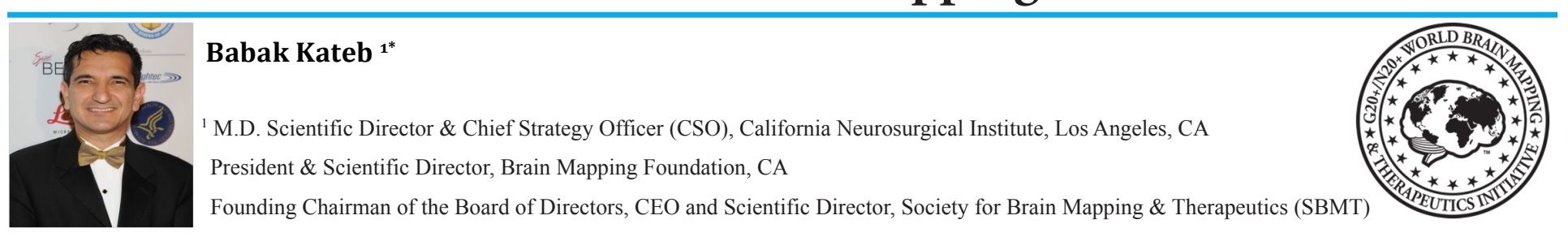

Chairman, G20+ World Brain Mapping and Therapeutics Initiative (G20+ WBMTI)

Director, National Center for NanoBioElectronics (NCNBE)

Visiting Scientist NASA/JPL, Pasadena, CA

Guest Editor, SPIE-SBMT Neurophotonic and Brain Mapping Journal

* Corresponding Author Address: 25751 McBean Parkway, Suite 305, Valencia, California 91355. Tel +1 (661) 799-2542, +1 (818) 827-2720, Fax +1 (661) 253-0248

Article Type: Editorial

Received: November 3, 2016, Last Revised: November 6, 2016, Accepted: November 9, 2016, Published: December 30, 2016

G20 World Brain Mapping Initiative (Neuroscience/N-20) is Putting Brain Mapping on the top of Global Economic Issues

The Society for Brain Mapping, Therapeutics, and Brain Mapping Foundation are propelling therapeutic advances in Nano-Neurosurgery, NanoBio-Electronics, Artificial Intelligence, Neuro-Supercomputing and 'cross-pollination' among the bio-medical sciences and engineering worldwide. “Think of Brain Mapping as a google map, the snapshot of earth being equivalent of the MRI. Now zoom down to the cities, which represents a Functional MRI, then zoom on the roads, which represent brain connectomics. If you zoom down further, you will see the cars, which represent brain cells and people in the car represent brain genomics and nanoscale structures" explains Dr. Babak Kateb, Founding Chairman of the Board of SBMT, Scientific Director of California Neurosurgical Institute.

Please cite this paper as: Kateb B. What Is Brain Mapping? Iran. J. Neurosurg. 2016;2(3):6-7

The science of brain mapping and the field of nanoneurosurgery have made notable advances in recent years due in part to the efforts of the Society for Brain Mapping \& Therapeutics (SBMT) and the Brain Mapping Foundation (BMF). The society and the foundation were launched as a collaboration among the NASA Jet Propulsion Laboratory, USC Keck School of Medicine and the California Institute of Technology, which are now a global powerhouse in brain research, innovation, and technology.

The Brain Mapping Foundation's main focus is to advance cross-pollination of ideas across the physical sciences, neuroscience and the biological sciences through conventions, publications and global initiatives and consortia. SBMT and BMF also seek to further establish and fund the National Center for NanoBioElectronics (NCNBE), to promote the rapid integration of nanotechnology, devices, imaging, artificial intelligence, super-computing, predictive modeling, immunotherapy, cellular and stem cell therapy with the specific aim of introducing advanced diagnostics and therapeutics.

Since 2004, both organizations have played a vital role in US BRAIN Initiative - the SBMT members and its board members had long advocated for and later advised the National Institutes of Health (NIH), The White Office Science Technology and Policy (OSTP), DARPA and NSF on the initiative - and the G20 + /Neuroscience20+ Brain Mapping and Therapeutics Initiative. Currently, SBMT focused on establishing the Global Alliance for Nano-Bio-Electronics through its G20+ Brain Mapping \& Therapeutics Initiative/Neuroscience-20 and shaping policies in the fields of translational neuroscience and pushing the boundaries of science and technology by introducing emerging fields to scientists, engineers, and physicians. For example in 2013 SBMT and BMF published the inaugural textbook of Nanoneuroscience and Nanoneurosurgery followed by 2016 textbook of Neurophotonics and Brain Mapping.

In this regard, BMF and SBMT have established significant ties with international partners across the G20 Summit members.

In 2016 (The third Annual) G20/N20 World Brain Mapping and Therapeutics gathering took place in the beautiful city of Chongqing, which led to the formation of SBMT-China. Drs. Yao Lu, Shu- Min Duan, Hau-Dong Zhou and Yan-Jian Wang are now taking the lead to further expand the SBMT China including new workshops and scientific meetings as well as publications and fellowships.

"The BMF's mission is to translate state-of-the-art technologies from NASA, National Labs, and industry into neurosurgery and neuroscience, to bring the most advanced medicine to patients with neurological disorders," Dr. Vicky Yamamoto, Cancer Scientist at USC-Keck School of Medicine and Executive Director of SBMT said. "The foundation is supporting basic and clinical scientists who are interested in brain mapping, engineering and nanoneurosurgery, and related areas, to improve patients' diagnosis, treatment, and rehabilitation."

The SBMT focuses principally on brain mapping (pre, per and postoperatively) and therapeutics including genomics, neurophotonics and nanoneurosurgery. SBMT pursues its mission through multi-disciplinary collaborations with government agencies, patient advocacy groups, educational institutes and industry, as well as philanthropic organizations. "The BMF supports the society's collaborative efforts and related activities both nationally and globally," Dr. Ken Green, Vice President of Brain Mapping Foundation.

The BMF also has humanitarian arm called: Global Physicians and Scientists (GPS), which aimed at mobilizing physicians, surgeons, and scientists to serve for a few weeks in poor, rural areas in the United States and abroad. GPS now uses SBMT's national and international centers and global network of near 120,000 scientists, engineers and physicians as their bases and networks of operations. 


\section{SBMT'S EDUCATIONAL FOCUS}

The society has been on the forefront of a broad educational agenda since its founding. Besides hosting world congresses, symposia and meetings, and advising on brain policy through its "Brain Mapping Day" at the US Congress, SBMT also offers fellowships, facilitates a visiting scholars program, and operates an awards program. The awards program identifies the most important scientific, clinical, engineering and policy contributions to the brain-mapping field. The SBMT presented Dr. Bennet Omalu, who discovered chronic traumatic encephalopathy in former football players, its Pioneer in Medicine award at its recent 2016 annual conference in Miami. The society has Beacon of Courage and Dedication award, which is aimed at scientists, patients and or patient family members who have shed light the neurological disorders with their leadership and heroism. SBMT strongly believe in educational outreach. One of the programs the Society is pushing forward includes student chapters and a K-12 neuroscience program to promote interest in brain mapping and therapeutics.

The society and the BMF are also active on the publishing front. The organizations supported a series of special issue publications in NeuroImage, a peer-reviewed journal covering pioneering and game-changing research on neuroimaging, nanoneurosurgery, robotics, neurophotonics, mathematical modeling/predictive modeling in cancer, supercomputing, functional neurosurgery. In 2010, The society established the first multidisciplinary journal in partnership with PLOS ONE called NeuroMapping \& Therapeutics Collection. BMF and SBMT also played a major role in publishing the inaugural Textbook of Nanoneuroscience and Nanoneurosurgery and the inaugural Textbook of Neurophotonics and Brain Mapping (CRC Press, Taylor, and Francis Group).

The BMF funds multidisciplinary translation research in several areas. In recent years, for example, the BMF has supported research on the potential for using NASA/UV Imaging cameras used to explore for supernova in the operating room, using NASA-JPL "Carbon-nanotubes" to advance personalized drug delivery to brain tumor, and using infrared thermography for intraoperative brain tumors mapping as well as advance neurophotonics for intraoperative tumor delineation. SBMT is one of the most collaborative organizations in the world. The organization has developed numerous partnerships and Joint CME sponsorship conventions and courses over the last decade. In addition to the AANS, the International Society for Magnetic Resonance Imaging in Medicine (ISMRM), the American College of Radiation Oncology (ACRO) and the National Space Biomedical Research Institute (NSBRI), among many others, SBMT has established relationships with more than 200 educational and research institutions worldwide. The organization also has established connections with an ever-growing list of biotech, pharmaceuticals, and device manufacturer (near 5000 of them worldwide).

The 3rd annual G20 World Brain Mapping \& Therapeutics Scientific Summit, held in Chongqing, China in 2016, included leading brain-mapping experts from the U.S, Australia, Japan, Turkey and the Middle East, as well as other G20 members' scientists, engineers, and physicians. Drs. Yao Lu, Shu- Min Duan, Hau-Dong Zhou and Yan-Jian Wang were the leading organizers.

For information about the initiative, visit www.worldbrainmapping.org. Membership details are available at http://www.worldbrainmapping.org/uncategorised/ membership. Additional questions may be directed to Dr. Vicky Yamamoto, SBMT’s executive director, at Vicky.Yamamoto@worldbrainmapping.org

"SBMT has been formulating global policies which could rapidly help scientists, integrate, translate and commercialize advanced diagnostics and therapeutics; we do have facilitated such interactions by bringing neurologists, neurosurgeons, neuroscientists, radiologist, engineers, cellular and molecular biologists, psychiatrists, physicist, nanotechnologists, stem cell scientists and oncologists together annually and globally"

Aaron Filler, MD, Ph.D

\section{HOW COULD SCIENTISTS, ENGINEERS, AND PHYSICIANS GET INVOLVED IN SBMT AND THE N20+ BRAIN MAPPING \& THERAPEUTICS INITIATIVE}

The society invites neurosurgeons and their colleagues to explore involvement opportunities in the SBMT's G20 World Brain Mapping Initiative. SBMT members, could participate in forming national and international symposia, be part of the science committee of the 2017 World Congress for Brain Mapping in Dubai, participate in 12 committees such as: CME, Science and technology, Credentialing, membership, Brain Policy and legislative affairs, help advocating advance funding support for translational medicine through our Brain Mapping Day at the US Congress, Parliaments of G20 Countries: Turkey, Canada and Australia. 